\title{
A Generalized Temporal Context Model for Semantic Scene Classification
}

\author{
Matthew Boutell \\ Department of Computer Science \\ University of Rochester \\ boutell@cs.rochester.edu
}

\author{
Jiebo Luo \\ Research and Development Laboratories \\ Eastman Kodak Company \\ jiebo.luo@kodak.com
}

\begin{abstract}
Semantic scene classification is an open problem in computer vision especially when information from only a single image is employed. In applications involving image collections, however, images are clustered sequentially, allowing surrounding images to be used as temporal context. We present a general probabilistic temporal context model in which the first-order Markov property is used to integrate content-based and temporal context cues. The model uses elapsed time-dependent transition probabilities between images to enforce the fact that images captured within a shorter period of time are more likely to be related. This model is generalized in that it allows arbitrary elapsed time between images, making it suitable for classifying image collections. We also derived a variant of this model to use in image collections for which no timestamp information is available, such as film scans. We applied the context models to two problems, achieving significant gains in accuracy in both cases. The two algorithms used to implement inference within the context model, Viterbi and belief propagation, yielded similar results.
\end{abstract}

\section{Introduction}

We see what we expect to see. The value of context for recognition has long been appreciated by various research communities. Temporal context is used in speech recognition: humans can understand phone conversations even when some of the syllables or words are muddled by noise, and all successful automatic speech recognizers use temporal context models. In computer vision, spatial context has been shown to improve object recognition $[16,19]$. In video processing, researchers also make strong use of temporal coherence for recognition purposes [8].

Because of a lack of context information, image understanding on still images has been recognized as more difficult than that on video streams [14]. However, image collections (e.g., photographs from film scans and digital camera downloads) represent a middle ground between the two. Each photograph is not captured in a vacuum, but between two other photos, giving it a temporal context. If the photos were captured hours apart, this context is probably meaningless, but if they were captured mere seconds apart, the content is likely to be related; digital cameras provide timestamps to distinguish the two situations.

In this paper, we develop and exploit temporal context models for the purpose of automatic scene classification of photographs. Automatically determining the semantic classification (e.g., mountain, sunset, indoor) of an arbitrary image has many useful applications, such as content-based image enhancement and organization [17].

We employ a two-stage approach, first classifying images solely using content embedded in pixels and then imposing a temporal context model (i.e., a Hidden Markov Model) consisting of entire image collections. We show that this model helps improve classification accuracy by correcting mistakes made by the content-based classifier, with little computational overhead. An advantage of this modular approach (over integrating the temporal constraints directly into the classifier, if possible) is that the temporal model can be used in conjunction with any content-based classifier.

In particular, we propose a general temporal context model that is a function of the elapsed time between images, such as can be derived from digital camera timestamps. When elapsed times are unavailable, as with film scans, this model degenerates to a special case, in which only the relative order of images is exploited. We confirm the intuition that using richer (elapsed-time) information increases performance more significantly. Further, we can treat video classification as a special case (with constant elapsed time between frames).

The main contributions of our work are a general (with respect to elapsed time), probabilistic temporal context model and its application to scene classification problems. This model is applicable to scenarios both with and without elapsed time. In addition, our algorithm does not rely on pre-clustering of the images into events, which would need to be done manually (labor intensive) or automatically (error prone [13]).

\subsection{Related work}

Semantic scene classification has been studied extensively in recent years (e.g., Refs. $[1,15,17,20]$ ). Most current systems use the low-level content (e.g., colors, 
textures, edges) of the image only and have achieved some success on constrained image sets (e.g., the Corel database). However, on unconstrained photographs, scene classification is still very much an open problem, especially when only image (e.g., pixel) information is used.

Meanwhile, relative time information (elapsed time between photographs) has been used successfully in two non-classification applications. First, clustering or grouping photographs by timestamps was used to complement content-based clustering strategies [6,13]. Second, Mulhem and Lim recently used the classification of images within a temporal cluster to improve image retrieval [9]. Their metric for relevance between a query $Q$ and a database image $\mathrm{D}$ incorporates both the match between $\mathrm{Q}$ and $\mathrm{D}$, but also the match between $\mathrm{Q}$ and the best-matching image in the same temporal cluster as D.

The approach proposed here is for classification, distinguishing it from the prior research in clustering [6] and retrieval [9]. While one could use clustering as a precursor to classification, this is not necessarily the best approach because clustering errors would propagate to the classification stage and degrade performance. Because our algorithm operates without the need for clustering, it also avoids the related overhead. In addition, our probabilistic approach differs greatly from the rule-based scheme in [9].

Recently, information from previous images was used to classify the current image in a video sequence [10]. We contrast our system with that work in the Conclusion.

\section{Probabilistic temporal context model}

In this study, we define an image "sequence" as a collection of photos captured in succession, but with arbitrary elapsed time between them (as would be the case with a typical amateur photographer's collection). We model image sequences using a Hidden Markov Model (HMM), representing the class of each image (a random variable) as a node in the graph and the temporal dependencies between nodes as edges (see Figure 1).

In image analysis, where the underlying content (e.g., classification) of a scene is to be inferred from an image, two independence assumptions are generally made $[2,4]$ : scene/observation dependence at each pixel or region, and locality (each scene node depends only on neighboring scene nodes). Our problem differs only in that our observations are for whole images, and the locality refers to dependence across neighboring images. However, the spirit of our assumptions is the same. First, the output of a content-based classifier on an image depends only on the class of that image and no other. Second, the class of an image depends only on the class of the images immediately preceding and succeeding it (its temporal neighborhood), and upon no other images. This locality assumption is the commonly known Markov property. The graphical model shown in

Figure 1 captures these assumptions.

We formalize the problem as follows: let $C_{i}$ be the true semantic classification (label) of image $i$, and $C=\left\{C_{l}, C_{2}\right.$, ..., $\left.C_{n}\right\}$ be a vector of the classifications of a sequence of $n$ images arranged in temporal order. The $1^{\text {st }}$-order Markov property can be stated formally as $P\left(C_{i} \mid\left\{C_{j}\right\}, j \neq i\right)=P\left(C_{i} \mid C_{i-1}, C_{i+1}\right)$. Let $M$ be the number of possible scene labels. Let $E_{i}$ be the evidence based on image $i$ alone for the class of image $i$, and $E=\left\{E_{1}, E_{2}, \ldots\right.$, $\left.E_{n}\right\}$ be the corresponding evidence vector. We call $E$ content-based evidence, because it is derived from the content (e.g., color, texture) of the image. In the terminology of HMMs, the classes are the (hidden) states and the evidence is the observed output.

Our goal is to maximize the probability of classifying the sequence of images correctly, given both the content-based evidence and the context relationships between images. In this formalism, our goal is:

$$
\begin{aligned}
\arg \max P(C \mid E) & =\quad \arg \max P(E \mid C) P(C) \\
& =\arg \max \left(\prod_{i=1}^{N} P\left(E_{i} \mid C_{i}\right)\right) P(C)
\end{aligned}
$$

The derivation is standard for HMMs [7], Bayes' rule followed by our first assumption above. We discuss learning $P\left(E_{i} \mid C_{i}\right)$ and $P(C)$ in Section 5.

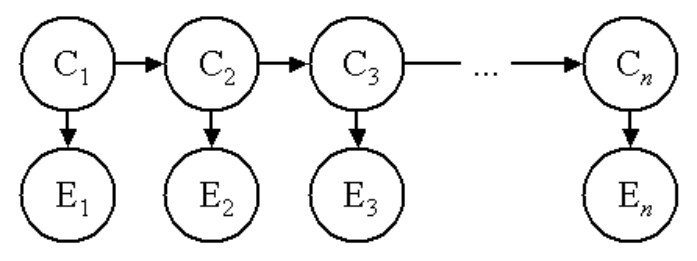

Figure 1. An appropriate graphical model for temporally related images is a Hidden Markov Model.

\section{Inference algorithms}

We now discuss two efficient inference schemes for use in HMMs, each with its own method of expanding and simplifying $P(C)$ using the Markov property.

\subsection{Viterbi inference algorithm}

In real-time speech recognition, one usually models the prior probability $P(C)$ using a bigram model (in which the context of each state consists of the previous state only). Using the chain rule successively and then applying the Markov Property for bigram models gives Equation 2. In the 
last line, we define $P\left(C_{l} \mid C_{0}\right)=P\left(C_{l}\right)$ for notational convenience.

$$
\begin{array}{ccc}
P(C) & = & P\left(C_{N} \mid C_{1} \ldots C_{N-1}\right) P\left(C_{1} \ldots C_{N-1}\right) \\
& =P\left(C_{N} \mid C_{1} \ldots C_{N-1}\right) P\left(C_{N-1} \mid C_{1} \ldots C_{N-2}\right) \ldots P\left(C_{2} \mid C_{1}\right) P\left(C_{1}\right) \\
& = & \prod_{i=1}^{N} P\left(C_{i} \mid C_{i-1}\right)
\end{array}
$$

To maximize Equations (1) and (2), a brute force algorithm requires complexity that is exponential in the number of images. However, because the conditional probabilities "flow" in one direction only, we may use the Viterbi algorithm, an efficient dynamic programming algorithm, to perform the optimization [3,7]. The Viterbi algorithm iterates forward through the list, keeping track of, for each state, the optimal path (maximal probability) to that state from the start, then backward, to read the optimal path. The efficiency is gained because the optimal path to any state $S_{i}$ must contain one of the optimal paths to state $S_{i-1}$, allowing local computations at each node [7].

\subsection{Belief propagation}

Markov networks, in the form of Markov Random Fields (MRFs), have been used in the computer vision community for problems of inferring scenes from images (e.g., [2,4]). However, the 2D grid used in those cases contains loops (as opposed to a simple chain), for which no exact efficient inference algorithms exist. Typical inference in this topology is done using greedy [2] or stochastic methods $[5,11]$. Belief propagation [4] is a message-passing algorithm that successfully ignores these loops in many graphs (and is exact in topologies without loops). It is a generalization of Pearl's algorithm for inference in Bayesian networks [12], in which messages passed in opposite directions do not interfere.

In belief propagation, each node (i.e., image in our study) calculates its own Maximum a Posteriori (MAP) estimate of the scene class. The factorization of $P(C)$ is different for each node; its derivation for node $i$ is as follows.

$$
\begin{aligned}
P(C) & =\quad P\left(C_{i}\right) P\left(C_{1} \ldots C_{i-1}, C_{i+1} \ldots C_{N} \mid C_{i}\right) \\
& =P\left(C_{i}\right) P\left(C_{1} \ldots C_{i-1} \mid C_{i}\right) P\left(C_{i+1} \ldots C_{N} \mid C_{i}\right) \\
& =P\left(C_{i}\right)\left(\prod_{k=2}^{i} P\left(C_{k-1} \mid C_{k}\right)\right)\left(\prod_{k=i}^{N-1} P\left(C_{k+1} \mid C_{k}\right)\right)
\end{aligned}
$$

This corresponds to the following message passing algorithm for the MAP estimate at node $j$ (specific to chain topology, see [4] for derivation of the general case). For chains, the MAP estimate is:

$$
\arg \max _{C_{j}} P\left(C_{j}\right) P\left(E_{j} \mid C_{j}\right) L_{(j-1) j}\left(C_{j}\right) L_{(j+1) j}\left(C_{j}\right)
$$

$P\left(C_{j}\right)$ is the prior on image $j, P\left(E_{i} \mid C_{i}\right)$ are the same output probabilities used in the Viterbi algorithm, and the likelihood messages $L_{k j}\left(C_{j}\right)$ from node $k$ to node $j$ about scene class $C_{j}$ are calculated using:

$L_{k j}\left(C_{j}\right)=\max _{C_{k}} P\left(C_{k} \mid C_{j}\right) P\left(E_{k} \mid C_{k}\right) \widetilde{L}_{l k}\left(C_{k}\right)$

where the $\tilde{L}$ are the messages from the previous iteration (initially set to 1 ) and node $l$ is node $k$ 's other neighbor. $E$ and $C$ in our notation correspond to $y$ and $x$, respectively, in the notation of [4]. Messages are passed until the MAP estimates converge. Because our topology contains no loops, it is guaranteed to converge to an exact solution in at most $N$ steps.

\section{Elapsed time-dependent transition probabilities}

The transition probabilities, $P\left(C_{i} \mid C_{j}\right)$, model temporal context between images. For example, if an image is both preceded by and succeeded by images of indoor scenes, we expect that it, too, is of an indoor scene. Transition probabilities govern the strength of class relationships between neighboring images. However, an integral part of this work rests on the following observation:

\section{"Transition strength between two images increases as the elapsed time between them decreases"}

For example, two adjacent images captured 17 seconds apart are almost guaranteed to be of the same event, while ones captured three weeks apart are probably unrelated. We formalize the fact that transition probabilities are dependent on elapsed time as follows. Let $\tau$ be the elapsed time between two neighboring images, and the probability distribution $\mathrm{e}(\tau)$ (e.g., exponential), be the prior on elapsed time. The class of an image is conditioned on the class of the previous image and the elapsed time between them: $P\left(C_{i} \mid C_{i-1}, \tau\right)$, where the $C_{i}$ are discrete and $\tau$ is continuous. This conditional distribution can be specified using $M(M-1)$ functions (free parameters), which we denote as transition functions. For example, consider the transition function $P\left(C_{i}=c \mid C_{i-1}=c, \tau\right)$, corresponding to adjacent images having the same class (Figure 2), e.g., $c=$ indoor. Ideally this function is expected to decrease monotonically (because the photographer can only move so fast) and approach the prior $P\left(C_{i}=c\right)$ in the limit as $e(\tau) \rightarrow \infty$. The transition functions are appropriate for use in situations with or without timestamps.

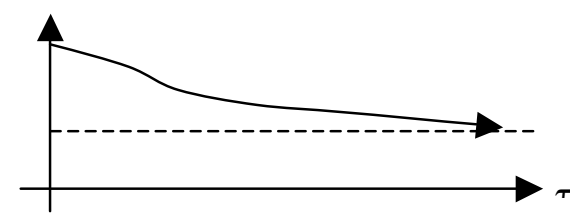

Figure 2. Transition function for $P\left(C_{i}=c \mid C_{i-1}=c, \tau\right)$. 


\section{Learning}

The graphical model requires two types of probabilities: transition probabilities and output probabilities. Each of these probabilities can be either set by an expert or learned from data. We chose to learn them from data (but understand that they are intuitive enough to be fine tuned by experts). We start with the transition probabilities, both dependent upon and independent of elapsed times.

\subsection{Elapsed time-dependent transition probabilities}

Each pair $(i, i+1)$ of adjacent images in a test sequence has an associated elapsed time $\tau_{i}$ between them. We have three choices regarding how to use $\tau_{i}$. The simplest is to discretize $\tau_{i}$ using discrete time slices. Another is to create these discrete slices and then interpolate between them. This method may be more precise if there is a large difference between transition probabilities in adjacent bins (assuming that the transition probabilities change smoothly over elapsed time). Third is to model the decrease in transition strength using a continuous function, helpful if the data is noisy. We chose the first option, due to its simplicity and faithfulness to the data.

We consider a discrete slice, $\mathrm{S}_{\mathrm{j}}=\left[\tau_{\mathrm{j}}, \tau_{\mathrm{j}+1}\right)$, of elapsed times (e.g., 5-15 minutes). To determine the transition probabilities for two images with elapsed times in S, we need to calculate the probability:

$$
\begin{aligned}
& P\left(C_{i} \mid C_{i-1}, \tau \in S_{j}\right)= \\
& \quad\left(\int_{\tau_{j}}^{\tau_{j+1}} P\left(C_{i} \mid C_{i-1}, \tau\right) e(\tau) d \tau\right) /\left(\int_{j}^{\tau_{j+1}} e(\tau) d \tau\right)
\end{aligned}
$$

Calculating E directly is nontrivial. A reasonable discrete approximation can be easily obtained from our training data by partitioning the image pairs in the training set according to elapsed time, counting the number of pairs in each partition, and then normalizing the frequencies to obtain the appropriate probabilities (e.g., Table 1). We used nonoverlapping slices that increased exponentially in length, mitigating the fact that the bins contain a monotonically decreasing number of images per bin. Note that indoor and outdoor probabilities are non-symmetric because they have different priors.

The discretized transition probabilities can be visualized as a 3-D matrix, in which the added dimension is elapsed time, $\tau$ (Figure 3). Each plane of the matrix is a 2-D matrix $P\left(C_{i} \mid C_{i-1}, \tau=\tau_{k}\right)$. As stated, the strength of the transitions is expected to drop as elapsed time increases, asymptotically approaching the prior probabilities of each class. The planes are not uniformly spaced with respect to $\tau$ for the reasons discussed above.

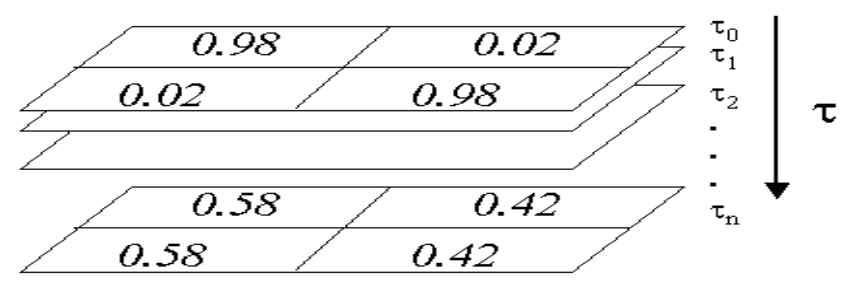

Figure 3. Elapsed time-dependent temporal context model. The transition probabilities used between two images are a function of the elapsed time between them. As $\tau \rightarrow \tau_{n}$, the probabilities approach the class priors.

\subsection{Marginalized transition probabilities}

When elapsed time information is unavailable, one needs to use the marginal probability over elapsed time. This is obtained by calculating the transition functions (Equation $7)$, over the whole elapsed-time domain $\left(S_{D}=[0, \infty)\right)$. This is equivalent to finding the average of the discrete slices $S_{i}$ (from Table 1), weighted by the proportion of training images in each slice (i.e., the probability distribution of training images over elapsed times):

$$
\begin{aligned}
& P\left(C_{i} \mid C_{i-1}, \tau \in S_{D}\right)=\sum_{j} N_{S_{j}} P\left(C_{i} \mid C_{i-1}, \tau \in S_{j}\right) \\
& \quad=\sum_{j} N_{S_{j}}\left(\int_{\mathbf{t}_{j}}^{\tau_{j+1}} P\left(C_{i} \mid C_{i-1}, \tau\right) e(\tau) d \tau\right) /\left(\int_{\mathbf{t}_{j}}^{\tau_{j+1}} e(\tau) d \tau\right) \\
& =\sum_{j}^{\tau_{j+1}} \int_{\tau_{j}} P\left(C_{i} \mid C_{i-1}, \tau\right) e(\tau) d \tau \\
& \quad=\int_{0}^{\infty} P\left(C_{i} \mid C_{i-1}, \tau\right) e(\tau) d \tau
\end{aligned}
$$

The proportion $N_{S j}$ is equal to the integral $\int_{\tau_{j}}^{\tau_{j+1}} e(\tau) d \tau$, causing the two to cancel in the second line of the derivation. The transition probability $P\left(C_{i} \mid C_{i-1}, \tau \in S_{D}\right)$ for the indoor-outdoor classification problem is shown in Table 2, obtained by finding a weighted average of the rows in Table 1. Conceptually, this is the same as the weighted average of the slices in Figure 3.

\subsection{Output probabilities}

Output probabilities $P\left(E_{i} \mid C_{i}\right)$ are relative likelihoods of observing the evidence, given the true scene class and relate to the output of the content-based classifier. Because an SVM classifier is binary and produces real-valued output, 
we shape the output by a sigmoid function [18] into a pseudo-probability that image $i$ belongs to class 1 . When computing the MAP solution, only the likelihood of the evidence is important. We view the SVM output as virtual evidence and use the same treatment as Pearl (see [12, p. 44]). In this case, we consider the content-based evidence to be uncertain or fuzzy input, with probability $P\left(E_{i}\right)$.

\section{Problem 1: Indoor-outdoor classification}

Our baseline content-based classifier is a Support Vector Machine using color and texture features, designed in a similar way to [17]. We trained it on an independent set of images not used elsewhere in this study.

Our image database consists of 24,000 pictures with a wide variety of image content; 56 photographers from three U.S. cities took pictures over the course of 12 months. We selected a subset, D1, of 132 manually-labeled image sequences randomly sampled from the database (containing a total of 2049 images). Of these, 1205 were indoor, giving a prior probability of $\mathrm{P}$ (indoor) $=0.588$.

We learned elapsed-time dependent transition probabilities from D1 (Table 1). The transition probabilities, $\mathrm{P}\left(\mathrm{C}_{\mathrm{i}} \mid \mathrm{C}_{\mathrm{i}+1}\right)$, in the other direction (needed for belief propagation) were close, but not identical, causing slight differences in performance of the two inference algorithms (Table 3). As expected, the strength of the probabilities diminished as the elapsed time between images increased. Because there were more indoor images than outdoor images, the probabilities are not symmetric. For cases where elapsed time is unavailable, we obtain the bigram transition probabilities shown in Table 2 through marginalization.

Table 1. Elapsed-time dependent transition probabilities learned from data set D1. Note the trend towards the prior probability as elapsed time increases.

\begin{tabular}{|l|l|l|l|}
\hline Time Interval & nImages & $\mathrm{P}\left(\mathrm{C}_{\mathrm{i}}=\mathrm{in} \mid \mathrm{C}_{\mathrm{i}-1}=\right.$ in $)$ & $\mathrm{P}\left(\mathrm{C}_{\mathrm{i}}=\right.$ out $\mid \mathrm{C}_{\mathrm{i}-1}=$ out $)$ \\
\hline $0-30 \mathrm{~s}$ & 1040 & 0.994 & 0.985 \\
\hline $30-90 \mathrm{~s}$ & 528 & 0.973 & 0.974 \\
\hline $90 \mathrm{~s}-5 \mathrm{~m}$ & 260 & 0.933 & 0.952 \\
\hline $5 \mathrm{~m}-15 \mathrm{~m}$ & 145 & 0.847 & 0.849 \\
\hline $15 \mathrm{~m}-45 \mathrm{~m}$ & 109 & 0.897 & 0.863 \\
\hline $45 \mathrm{~m}-2 \mathrm{~h}$ & 98 & 0.803 & 0.757 \\
\hline $2 \mathrm{~h}-6 \mathrm{~h}$ & 62 & 0.697 & 0.621 \\
\hline $6+\mathrm{h}$ & 314 & 0.707 & 0.455 \\
\hline
\end{tabular}

We evaluated the system performance on D1 as well, mainly because of a shortage of labeled data. This is not a concern. First, we had trained our content-based SVM classifier on a completely independent set of images. Second, training and testing on the same set of images would be a concern for classifiers that discover patterns (e.g. SVM) and can therefore "memorize" data sets.
However, to facilitate the temporal context model, we are merely deriving simple statistics (state transition probabilities) from the data. We confirmed that our algorithm is not sensitive to small perturbations in these simple statistics.

For testing, each image was classified by the contentbased classifier, transition probabilities were calculated based on elapsed time when applicable, and then a temporal inference algorithm (Viterbi or belief propagation) was executed on each sequence. Accuracy is shown in Table 3.

Table 2. Transition probabilities learned from data set D1, marginalizing over elapsed time, for the order-only case.

\begin{tabular}{|l|l|l|}
\hline \multirow{2}{*}{$\mathrm{C}_{\mathrm{i}-1}$} & \multicolumn{2}{|c|}{$\mathrm{C}_{\mathrm{i}}$} \\
\cline { 2 - 3 } & Indoor & Outdoor \\
\hline Indoor & 0.924 & 0.076 \\
\hline Outdoor & 0.099 & 0.901 \\
\hline
\end{tabular}

Table 3. Accuracy of the order-only and elapsed time context models using both inference schemes.

\begin{tabular}{|l|l|l|}
\hline \multirow{2}{*}{ Context Model } & \multicolumn{2}{|c|}{ Inference Method } \\
\cline { 2 - 3 } & Viterbi & Belief Propagation \\
\hline None & \multicolumn{2}{|c|}{$81.0 \%$} \\
\hline Elapsed Time & $86.8 \%$ & $86.5 \%$ \\
\hline Order only & $85.9 \%$ & $85.5 \%$ \\
\hline
\end{tabular}

The two inference algorithms performed approximately the same, with the small difference due to data dependency, as described above. Both temporal context models clearly outperformed the baseline, more significantly with the elapsed time-dependent model. This is mainly because relations between classes of images captured days apart were (rightfully) not enforced. Figure 4 shows the ROC curves for the baseline and elapsed-time temporal model using Viterbi inference (obtained by varying the bias of the baseline classifier).

Our probabilistic temporal context models boosted accuracy by correcting a large number of images classified incorrectly by the baseline content-based classifier. At the same time, it also caused very few images to be classified incorrectly. Figure 6 shows examples of images for which the context model changed its classification. The first two sequences are examples in which the temporal model helped correct the baseline classifier's errors. In each case, if one views each image in isolation, it is understandable why the color-texture classifier made errors. However, the short elapsed time between images made the baseline classifier's predictions unlikely. Conversely, the model biased against the rare cases in which the photographer moved from outdoors to indoors and back again within seconds (e.g., 
sequence 3 ), but such errors are a small price to pay for the gains the model makes. The last sequence shows a misclassification that the model cannot correct because the image was captured in relative isolation (between two temporally-distinct events).

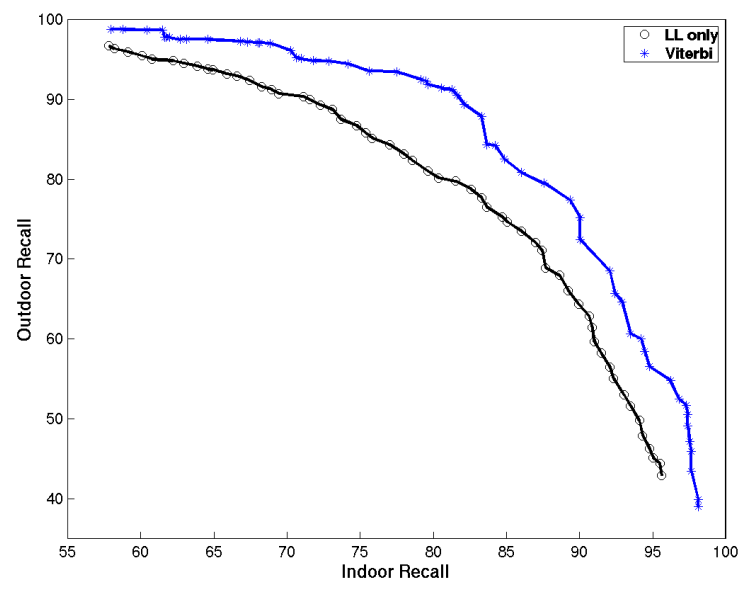

Figure 4. Comparison of the baseline content-based indooroutdoor classifier with that enhanced with the elapsed-time temporal model. Note that this is not a typical ROC curve because we want to show the balance between accuracy on each of the two classes.

\section{Problem 2: Sunset detection}

To further demonstrate the efficacy of the context model, we also applied it to the problem of sunset detection (we use sunset generically to refer to both sunset and sunrise because most twilight scenes are sunsets). Similar to [1], our low-level classifier was a Support Vector Machine using spatial color moment features trained on an independent set of images unrelated to this study. For testing data, we used a collection of 4678 personal images containing 191 (4\%) sunsets. Figure 5 compares the performance of the contentonly classifier to that with the context model. The benefit is clear.

With a problem such as sunset detection, in which there are many more non-sunsets than sunsets, an operating point with low recall must often be used to keep a small false positive rate. However, the primary benefit of the context model is that for any threshold, many false positives can be rejected, allowing us to bias the baseline detector in favor of sunsets in order to increase the recall while holding a constant false positive rate. Figure 7 shows examples of gains that the context model can make. The first sequence is very typical. Indoor scenes under low incandescent lighting can be mistaken for sunsets by the color-texture classifier. However, those occurring between images with different lighting can easily be corrected. The second sequence shows sunsets with weak colors that were initially missed by the color-texture classifier but were eventually recovered when the context model was used.

Interestingly, from the examples shown for both applications, the classifier's error characteristics are different than those of the content-only classifier. Images classified in isolation (based on content) tend to have isolated errors, while the context model can cause errors to cluster. Arguably, in a semi-automatic system (e.g., desktop image albuming software), these errors would be more convenient for a human to correct.

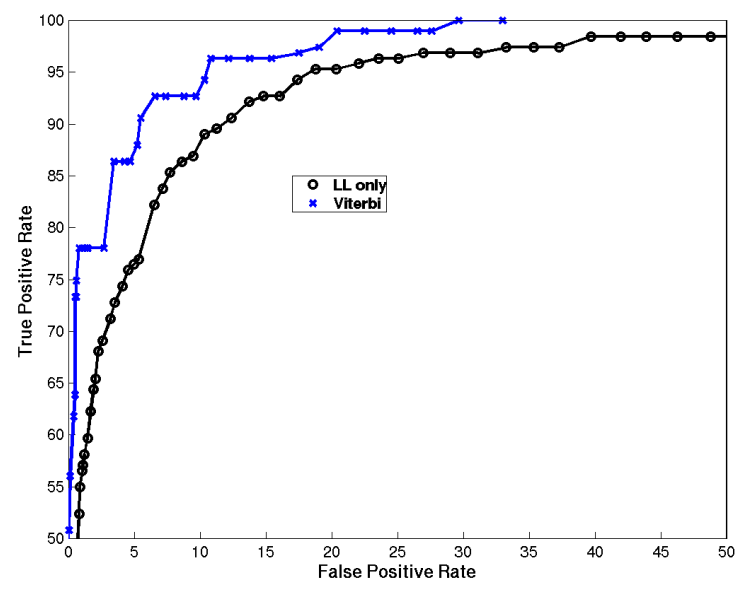

Figure 5. Comparison of baseline content-based sunset detector performance with that of the model. For any false positive rate, the recall of sunsets can be boosted by $2-10 \%$. Alternately, for a given recall rate, the false positive rate can be reduced by as much as $20 \%$ in high recall operating points.

\section{Conclusion and future work}

In this paper, we have proposed a general probabilistic temporal context model for scene classification, which exploits temporal coherence between neighboring images, especially if captured within a short length of time. The loopless topology of the model allows both exact and efficient calculation of the MAP solution, adding little overhead to the baseline content-based classifiers. The context model is also flexible, allowing for both images with and without timestamps. We have demonstrated the efficacy of the model on a pair of two-class scene classification problems: indoor-outdoor and sunset detection.

This work is related to [10] but represents a more generalized model for temporal context. While temporal context information was propagated to help classify scenes in [10], each scene was captured at a fixed frame rate in a video sequence. Our model is built upon elapsed-time dependent transition probabilities, allowing for general 
usage on unconstrained image collections with and without timestamps; video is essentially a special case where the elapsed time is constant.

In the future, we plan to extend our system to handle multi-class problems such as general outdoor scene classification or image orientation detection.

Second, we have only exploited the first-order Markov property in this work. However, local interactions can lead to very long (incorrect) runs of images of the same class, even when evidence is propagated along the sequence. To address this, we could exploit the second-order Markov property, i.e., approximating $\mathrm{P}\left(\mathrm{C}_{\mathrm{i}} \mid \mathrm{C}_{1}, \ldots \mathrm{C}_{\mathrm{i}-1}\right)$ with $\mathrm{P}\left(\mathrm{C}_{\mathrm{i}} \mid \mathrm{C}_{\mathrm{i}-2}\right.$, $\left.\mathrm{C}_{\mathrm{i}-1}\right)$. Preliminary results show that while the transition likelihood ratios for the marginalized first-order model are approximately $12: 1$, the ratios for the second-order model change to either $35: 1$ or $5: 1$, depending on whether the two previous classes agree or disagree. Alternately, we could investigate how to incorporate the expected run length of scene labels into the classifier.

\section{Acknowledgments}

We thank Prof. Christopher Brown (Univ. of Rochester) and Prof. William Freeman (MIT) for valuable discussions.

\section{References}

1. M. Boutell, J. Luo, and R.T. Gray. Sunset scene classification using simulated image recomposition. Proceedings of International Conference on Multimedia and Expo, 2003.

2. P. Chou. The Theory and Practice of Bayesian Image Labeling. PhD thesis, University of Rochester, Rochester, NY, 1988.

3. R. Duda, R. Hart, and D. Stork. Pattern Classification, $2^{\text {nd }}$ Edition. John Wiley and Sons, Inc., New York, 2001 .

4. W.T. Freeman, E.C. Pasztor, and O.T. Carmichael. Learning low-level vision. International Journal of Computer Vision, 40(1):24-57, October 2000.

5. S. Geman and D. Geman. Stochastic relaxation, Gibbs distributions, and the Bayesian restoration of images. IEEE Transactions on Pattern Analysis and Machine Intelligence, 6(6):721-741, November 1984.

6. A.C. Loui and A. Savakis. Automatic event segmentation and quality screening for albuming applications. Proceedings of IEEE International Conference on Multimedia Expo, 2000.

7. C. Manning and H. Schutze. Foundations of Statistical Natural Language Processing. MIT Press, Cambridge, MA, 1999.
8. D. Moore, I. Essa, and M. Hayes III. Exploiting Human Actions and Object Context for Recognition Tasks, Proceedings of International Conference on Computer Vision, 1999.

9. P. Mulhem and J-H Lim. Home photo retrieval: time matters. Lecture Notes in Computer Science, 2728:321330. 2003.

10. A. Torralba, K. Murphy, W. Freeman, and M. Rubin. Context-based Vision System for Place and Object Recognition. Proceedings of International Conference on Computer Vision, 2003.

11. R. M. Neal. Probabilistic inference using Markov Chain Monte Carlo methods. Technical Report CRG-TR-93-1, Dept. of Computer Science, University of Toronto, 1993.

12. J. Pearl. Probabilistic Reasoning in Intelligent Systems. Morgan Kaufmann Publishers, 1988.

13. J. Platt. AutoAlbum: Clustering digital photographs using probabilistic model merging. Proceedings of IEEE Workshop on Content-based Access of Image and Video Libraries, 2000.

14. N. Sebe, M. Lew, X. Zhou, T. Huang, and E. Bakker. The state of the art in image and video retrieval. Proceedings of International Conference on Image and Video Retrieval. 2003.

15. Y. Song and A. Zhang. Analyzing scenery images by monotonic tree. ACM Multimedia Systems Journal, 2002.

16. A. Singhal, J. Luo, and W. Zhu. Probabilistic spatial context models for scene content understanding, Proceedings of International Conference on Computer Vision and Pattern Recognition, 2003.

17. M. Szummer and R Picard. Indoor-outdoor image classification. Proceedings of IEEE International Workshop on Content-based Access of Image and Video Databases, 1998.

18. D. Tax and R. Duin. Using two-class classifiers for multi-class classification. Proceedings of International Conference on Pattern Recognition, 2002.

19. A. Torralba and P. Sinha. Statistical context priming for object detection. Proceedings of International Conference on Computer Vision, 2001.

20. A.Vailaya, M.Figueiredo, A.Jain, and H.-J. Zhang. Content-based hierarchical classification of vacation images. Proceedings of International Conference on Multimedia Computing and Systems, 1999. 


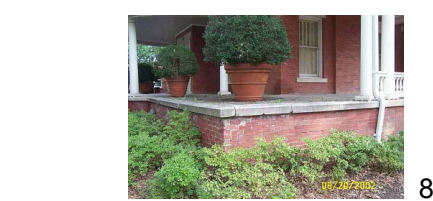

Baseline:

Model:

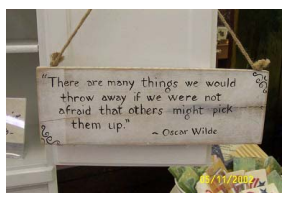

Bs:

I

Model:

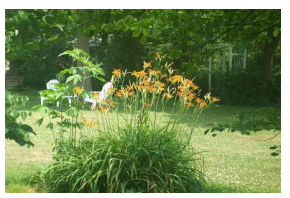

Bs:

Model:

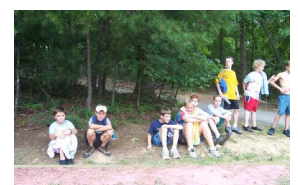

Bs:

0

Model:

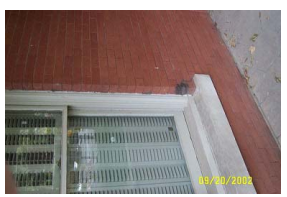

I

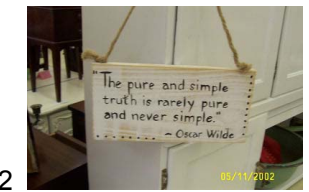

I
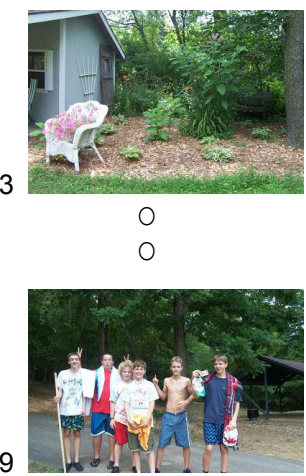

O

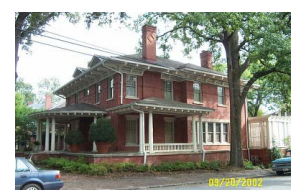

O

0

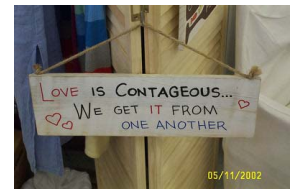

I

I

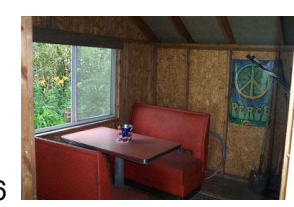

I

O

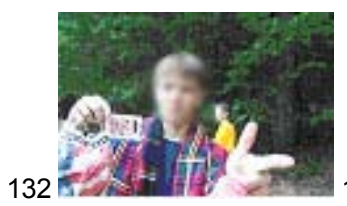

0

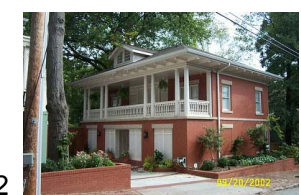

O

O

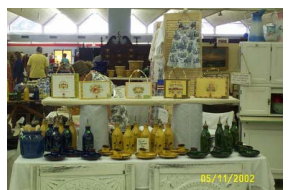

I

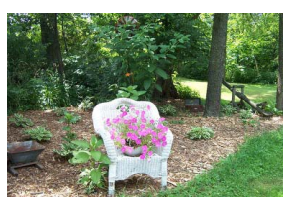

0

O

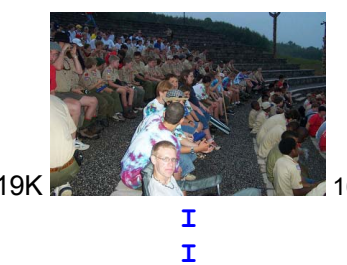

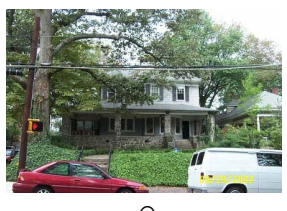

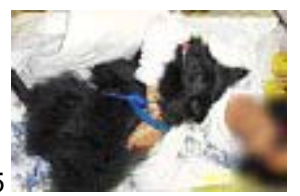

I

I

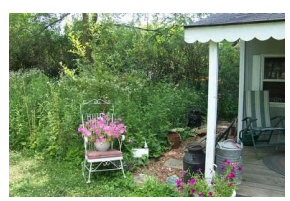

$\mathrm{O}$

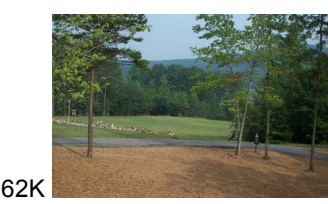

O

Figure 6. Image sequences affected by the context model. Elapsed times (in seconds) between images are shown. The first two sequences show examples in which the model corrected errors made by the baseline classifier. The third sequence shows a conceptual error: a rare case where the photographer walks into a room briefly between taking two outdoor photos.
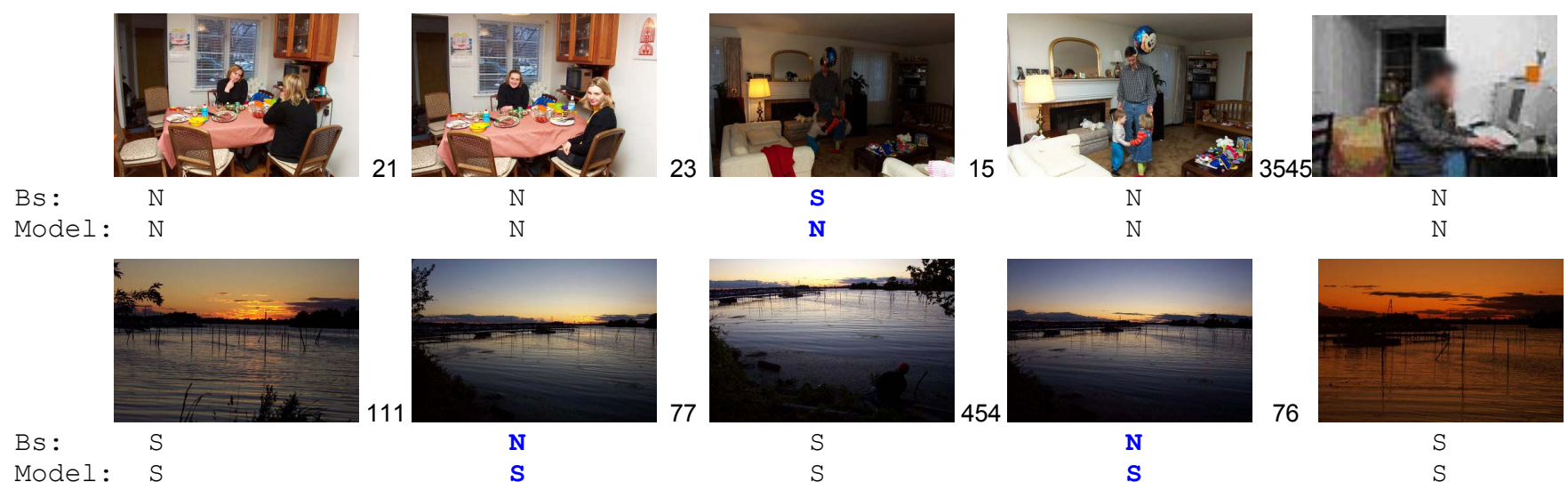

Figure 7. Two sunset image sequences affected by the context model. In each case, the model corrects an error. The first sequence is typical: indoor images under low incandescent lighting can often be confused as sunsets, but are easy to correct by the temporal model. The second sequence shows a burst of sunset images in which two "weak" (cool-colored) sunsets are missed by the color-texture classifier, but corrected by the model. 